\title{
Die pedocrurale Tinea bei vorgeschädigter Unterschenkelhaut und beim Ulcus cruris Die pedocrurale mykotische Infektionseinheit
}

\author{
Pedocrural Tinea in Previously Damaged Lower Leg Skin or Leg Ulcers \\ The Pedocrural Mycotic Infectious Unit
}

Autor

Institut

\section{P. Foss}

Hautarzt/Wundarzt, Wadern
Bibliografie

DOI 10.1055/s-0028-1119692

Online-Publikation: 8.5.2009

Akt Dermatol 2009; 35:

447-452 @ Georg Thieme

Verlag KG Stuttgart · New York

ISSN 0340-2541

Korrespondenzadresse Dr. med. Pierre Foss

Hautarzt und Wundarzt Kräwigstraße 8-10 66687 Wadern

pierre.foss@ana-podo.de

\section{Zusammenfassung \\ $\nabla$}

Die Arbeit beschreibt, wie die Onychomykose, Zehenzwischenraummykose oder Tinea pedis die Behandlung der chronisch venös-lymphatischen Stauungsdermatose mit Ulzerationen gefährden können. Die Tinea pedis, Zehenzwischenraummykose und die Onychomykose führen oft zu direkten mykotischen Infektionen der vorgeschädigten Haut des Unterschenkels und dortiger, chronischer Wunden. Diese Tinea cruris und Wundmykose zeigt aufgrund der Vorschäden nicht die klassischen klinischen Befunde der Tinea corporis und ist deshalb äußerlich schwer zu erkennen (Tinea incognita). Vor allem bei venös-lymphatisch bedingten chronischen Ulcera crurum, die in der Wundumgebung erosiv-nässende Hautverhältnisse aufweisen, ist das ideale Milieu für diese Nachbarschaftsmykose vorhanden. Die Diagnose sollte zytologisch oder über eine diagnostische Curettage histologisch gesichert werden. Die intensive antimykotische Lokaltherapie muss den gesamten Fuß und Unterschenkel als mykotische Infektionseinheit (pedocrurale Tinea) erfassen. Die konsequente antimykotische Lokalbehandlung bessert innerhalb weniger Tage den Hautzustand des Unterschenkels und erleichtert das moderne Wundmanagement. Bei Onychomykose und Tinea pedis sollte prophylaktisch die vorgeschädigte Unterschenkelhaut antimykotisch behandelt werden.

\section{Einleitung \\ $\nabla$}

Diese Publikation soll in Ergänzung zur Erstveröffentlichung der Wundgrundzytologie [3] den Fokus nicht nur auf die chronische Wunde, sondern den Blick auf die die Wunde umgebende Unterschenkelhaut lenken. Während bei der Wundgrundzytologie die bakterielle Schnelldiagnostik im Vordergrund stand, wird ins Zentrum dieser Veröffentlichung die mykotische Infektion der Wundumgebung und der chronischen Wunde als Nachbarschaftsmykose durch eine nachgewiesene Onychomykose und/oder Tinea pedis gestellt. Der Autor führt seit über 10 Jahren zytologische Untersuchungen zur Pilzdetektion der Haut und Nägel durch. Zudem betreut er schwerpunktmäßig Patienten mit chronischen Wunden, vor allem an den Unterschenkeln und Füßen, in seiner Praxis. Diese langjährigen Erfahrungen wurden genutzt, um gezielt Pilzinfektionen der vorgeschädigten Unterschenkelhaut und in chronischen Wunden nachzuweisen. Es wurden vor allem jene Ulkuspatienten der Praxis ausgewählt und intensiv abgeprüft, die ungeklär- te Beläge und Hautirritationen der Unterschenkelhaut aufwiesen.

\section{Material und Methodik \\ $\nabla$}

Von Mai 2008 bis Ende November 2008 wurden 30 Patienten mit chronischen Wunden und Stauungsdermatosen auf Tinea pedis und Onychomykosen überprüft. 18 Patienten wiesen eine Tinea pedis und/oder eine Onychomykose auf. Wiederum zeigten hiervon 8 Patienten an den Unterschenkeln Rötungen, Beläge, Schuppungen und Juckreiz, die nachweislich mykotisch bedingt waren. 6 Patienten hatten Pilzinfektionen in den Belägen der Unterschenkelhaut und 2 Patienten in den Wunden. Der Beweis der Pilzinfektion in der vorgeschädigten Haut der Unterschenkel (oft in der Umgebung der Wunden) und der Wunden selbst wurde zytologisch durchgeführt. Es wurden jeweils an mehreren Hautstellen des Unterschenkels zytologische Proben mittels Tesa-Film, Ringkürette oder einer gynäkologischen Rundbürste entnommen (Technik wie in ( $\mathbf{O} \mathbf{A b b} \mathbf{1})$. 


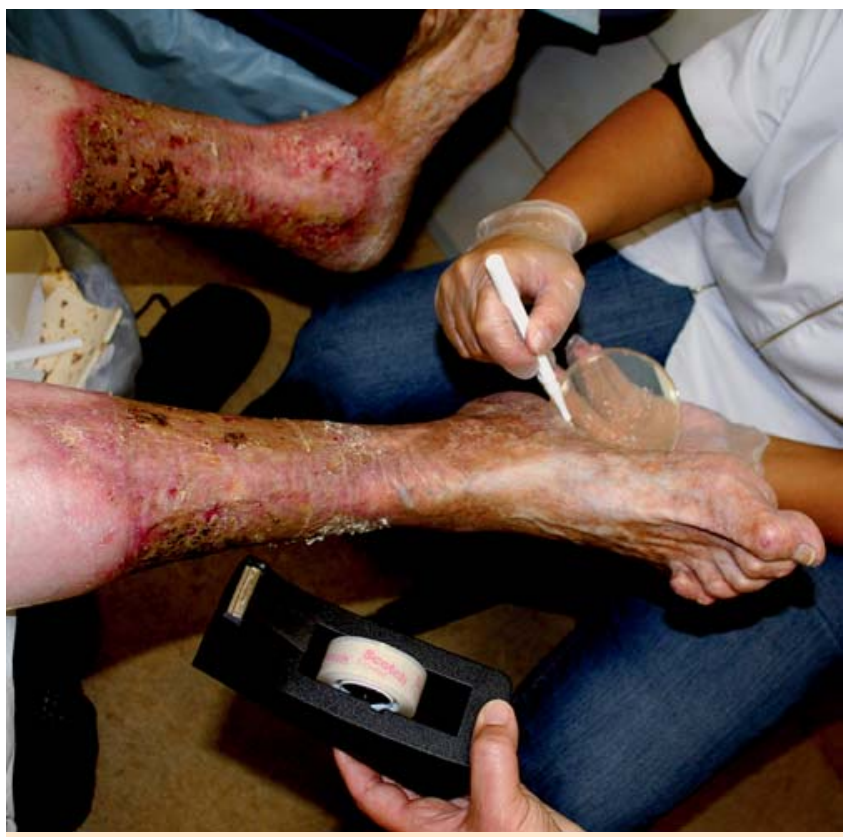

Abb. 1 Patient, 64 Jahre, Postthrombotisches Syndrom beidseits, Z.n. Hauttransplantationen. Seit 10 Jahren rezidivierende, venös bedingte Ulzerationen an beiden Unterschenkeln. Seit Jahren Beläge an den Unterschenkeln. Onychomykose und Tinea pedis nachgewiesen. Mykologische Diagnostik positiv durch Zytologie und Histologie mittels Tesa-Film und Ringcurette. Das klinische Bild ist nicht typisch für eine Tinea corporis. Kulturell wurde Candida parapsilosis und T. rubrum in den Belägen identifiziert und in den Nägeln T. rubrum.

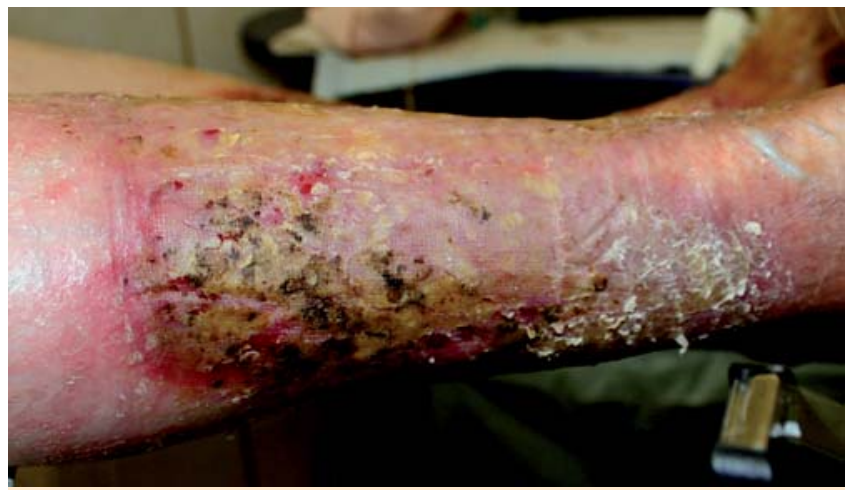

Abb. 2 Rechter Unterschenkel außen des gleichen Patienten wie in - Abb. 1. Erosiv nässende Stauungsdermatose mit Dermatoliposklerose und dicken, nässend-erosiven Belägen. In diesen Belägen wurden zytologisch Hyphennester gefunden. Untypisches klinisches Bild für eine Tinea corporis bzw. cruris. Die venös-lymphatische, chronische Vorschädigung (der Basisschaden, [4]) der Unterschenkelhaut dominiert das klinische Bild.

Nach dem Aufbringen auf einen Objektträger wurden die Proben mit Methylenblau oder nach PAS gefärbt und unter einem Lichtmikroskop mit 16-100× Objektivvergrößerung analysiert. Mikroskopische Bilder der beschriebenen zytologischen Befunde konnten nicht angefertigt werden, da kein Photomikroskop zur Verfügung stand und das Hauptaugenmerk der Publikation auf die klinischen Bilder der Befunde gelegt wurde. Dreimal wurden von den Hautbelägen zur weiteren Absicherung histologische Untersuchungen veranlasst, die die zytologischen Ergebnisse bestätigten. Das Material wurden mit Hilfe von Ringcuretten gewonnen. Kulturell wurde einmal Candida parapsilosis und zweimal Trichophyton rubrum identifiziert. Die restliche Kultur-

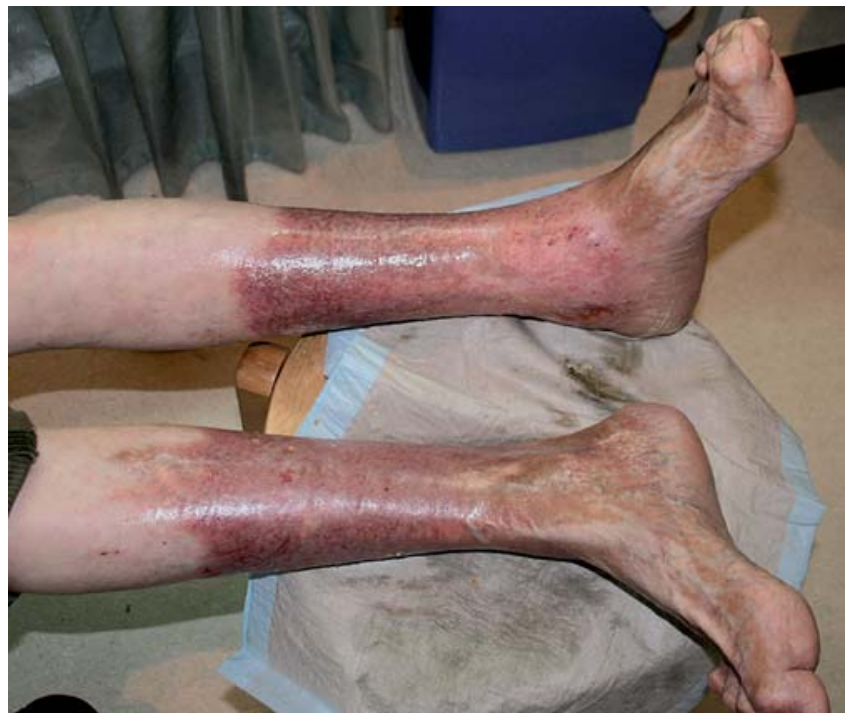

Abb. 3 Zustand nach 2 Wochen intensiver antimykotischer Lokaltherapie der pedocruralen Infektionseinheit mit Ciclopirox-Creme $1 \times$ tgl. und oral Fluconazol.

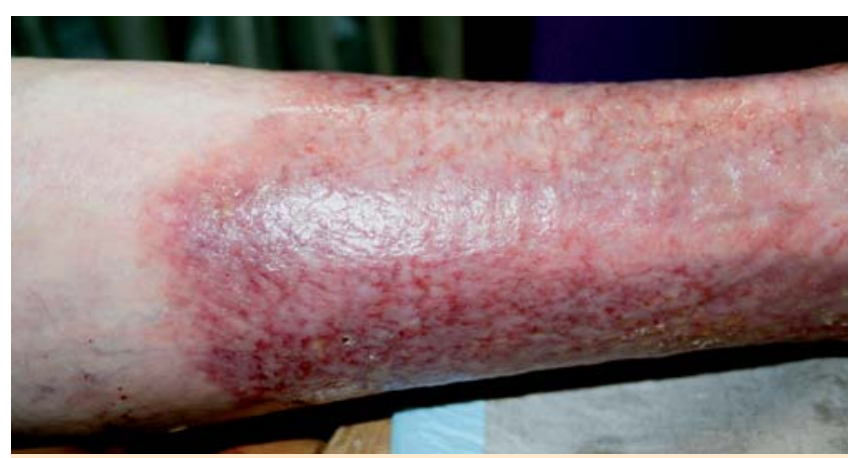

Abb. 4 Rechter Unterschenkel Detailansicht von $\oslash$ Abb. 3. Die Beläge sind verschwunden. Der Juckreiz ist abgeheilt, die Erosionen und die Entzündung deutlich gebessert.

diagnostik war negativ bzw. nicht auswertbar. Die Onychomykose respektive Tinea pedis wurden jeweils zytologisch verifiziert. In der Kultur konnte zweimal als Erreger Trichophyton rubrum und mentagrophytes identifiziert werden.

\section{Therapie}

Nach der Sicherung der Tinea cruris, die wahrscheinlich durch die Onychomykose und Tinea pedis des Patienten verursacht wurde, erfolgte eine konsequente lokale Anwendung von modernen breitbandantimykotischen Cremen (Ciclopirox, Terbinafin, Sertoconazol) $1 \times$ tgl., wobei der gesamte Fuß mit den Zehenzwischenräumen und der gesamte Unterschenkel eingecremt wurde. Zusätzlich wurden die Zehennägel mit antimykotischen Lösungen oder antimykotisch wirksamen Lacken behandelt. Die modernen Lokalantimykotika erfassen bekanntlich das gesamte relevante mykotische Keimspektrum und können deshalb bei richtiger Anwendung eine umfassende Therapiewirkung garantieren. Bei einem Patienten ( $\bullet$ Abb. 1-4) wurde nach gelungener Kulturanzucht oral Fluconazol und bei einem anderen oral kurzzeitig Terbinafin ( $\bullet$ Abb.12-16) eingesetzt. Unter dieser Therapiestrategie kam es innerhalb weniger Tage zu erheblichen 


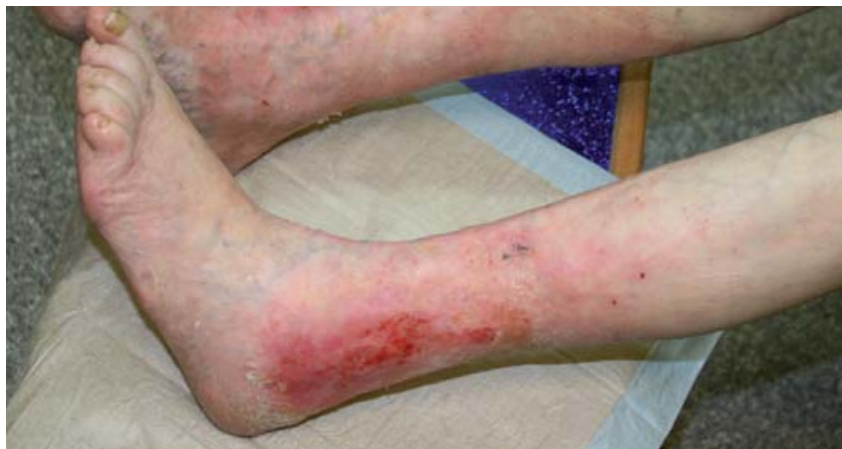

Abb. 5 77-jährige Patientin mit Ulzera mixtum an beiden Unterschenkeln, Onychomykose und Tinea pedis. Rötungen und dünne Beläge, Juckreiz.

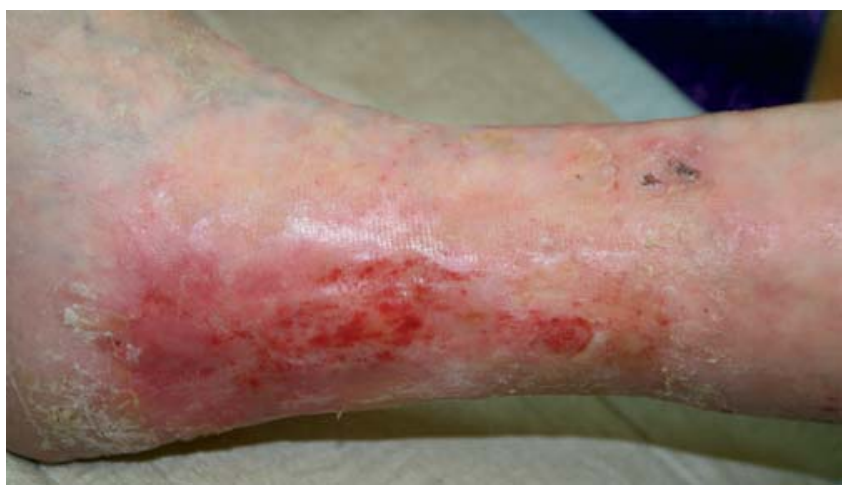

Abb. 6 Detailausschnitt von $\bullet$ Abb. $\mathbf{5}$ erosiv-nässende Haut am Außenknöchel, Dermatoliposklerose. Zytologie mittels Tesafilm in den Erosionen: Nester mit septierten Hyphenfäden.

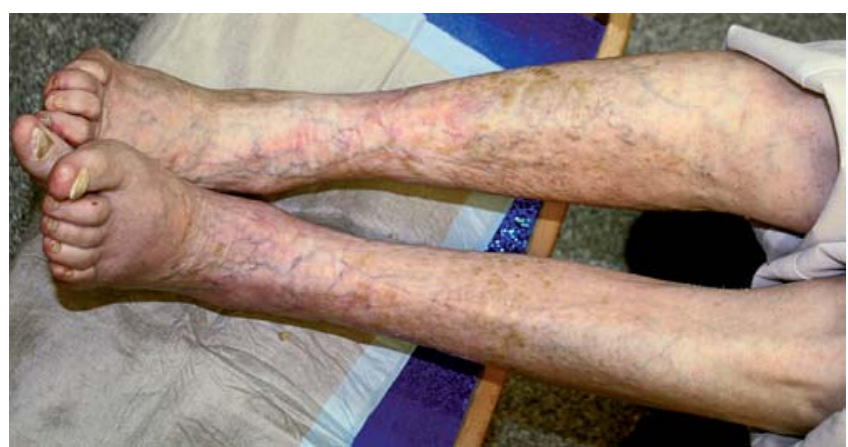

Abb. 7 Patientin nach 3 Wochen intensiver lokaler Antimykose mit Ciclopirox Creme, $1 \times$ tgl. Die Rötung und Beläge sind erheblich reduziert. Kein Juckreiz mehr.

Besserungen der Unterschenkelhaut bzw. der Wunden, die sich dann weiter normalisierten bis zu einem Zustand, der durch die Stauungsdermatosen bedingt war $(\bullet$ Abb. 3, 4, 7, 8, 16).

\section{Diskussion}

\section{$\nabla$}

Diese Publikation soll Anregung sein bei Onychomykosen, Tinea interdigitalis und Tinea pedis genau hinzusehen bzw. intensiv nach einer Pilzinfektion der Unterschenkelhaut zu suchen, wenn sich Rötungen, Juckreiz und Beläge des Unterschenkels zeigen, und nicht den Nagel- und Fußpilz als Bagatelldiagnose abzutun. Die Gefahr mykotisch bedingte Hautveränderungen

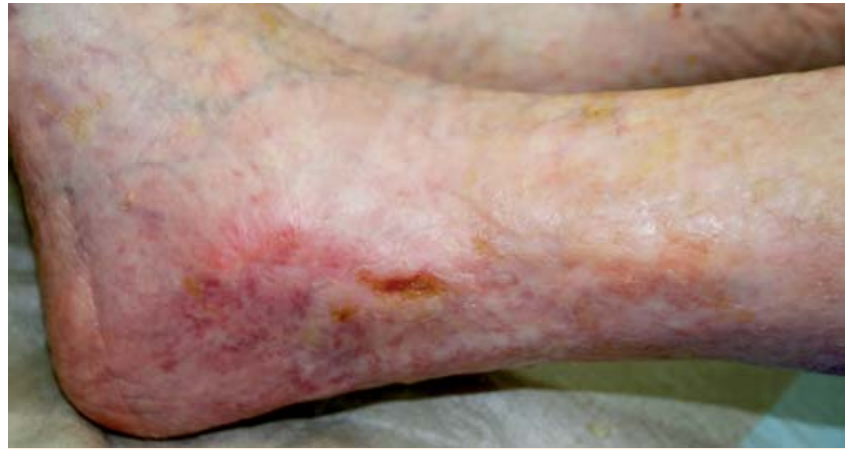

Abb. 8 Therapierter Zustand nach intensiver lokaler Antimykose der Patientin in Abb. 7. Erhebliche Besserung des Hautbefundes.

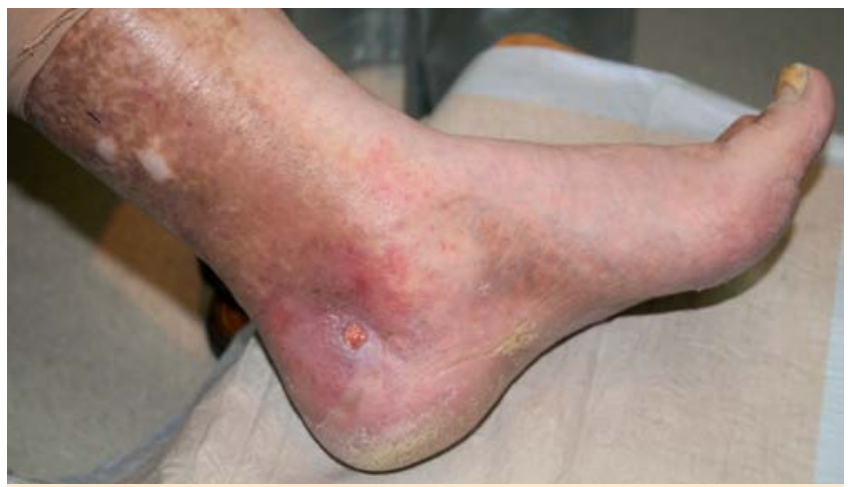

Abb. 9 Venöses Stauungssyndrom am Unterschenkel mit kleinem Ulkus am linken Innenknöchel. Onychomykose und Tinea pedis.

Im Belag des Wundgrundes Hyphennetze. Erhebliche Besserung des Ulkus bis auf Stecknadelkopfgröße unter konsequenter antimykotischer Lokaltherapie der pedocruralen Infektionseinheit einschließlich der Wunde mit einer Sertoconazol-Creme, $1 \times$ tgl.

auf vorgeschädigter Unterschenkelhaut falsch zu interpretieren ist groß, vor allem, wenn keine Vorortzytologie betrieben wird. Gleiches gilt für die mögliche Pilzinfektion der chronischen Wunde. Irritative und kontaktallergische Dermatitiden, Dermatosen bei Diabetes, Erysipele, Stauungsdermatosen und natürlich bakterielle Infektionen gehören zu den wichtigen Differenzialdiagnosen. Das klinische Bild zeigt nicht die üblichen Merkmale einer Tinea corporis $[1,2,4-8]$ mit den typischen anulären, randbetonten Rötungen. Das klinische Bild ähnelt an seltenen Hautstellen den Dermatophytosen der Extremitäten, wie sie in [2] umfassend beschrieben wurden. Die dort beschriebene diskontinuierliche Ausdehnung der Pilzherde am Unterschenkel wurde auch von mir beobachtet ( $\bullet$ Abb. 1, 2, 5, 6). Die Tinea auf einer Stauungsdermatose ist maskiert und bekommt eine erhebliche „Starthilfe durch den Basisschaden“ ( $\bullet$ Abb. 1, 2, 4-6) [4]. Die Wundmykose zeigt keinerlei spezifische Merkmale. Die Pilze finden in den feuchten Belägen der chronischen Wunden ein ideales Anzuchtmilieu ( $\bullet$ Abb.9-11).

Die Pilzsuche muss großflächig und an mehreren Stellen des Unterschenkels bzw. in den Belägen erfolgen, da die Hyphen bzw. Sporen dort sehr locker verteilt und oft in Nestern auf der Haut vorhanden sind. Oft zeigen sich wegen der Vorbehandlungen nur einzelne Sporenhaufen und Hyphen. Diese Pilzelemente sind zudem meist kleiner und dünner als üblich und müssen aufmerksam in den Präparaten gesucht werden. Die großflächige Probennahme ist auch unbedingt bei der Kulturabnahme ein- 


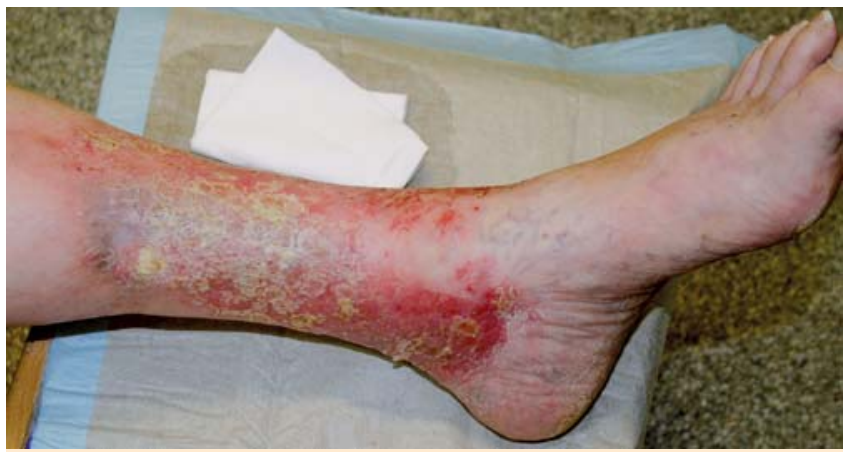

Abb. 10 68-jährige Patientin mit chronisch-venösem Stauungsekzem, rezidivierenden Ulzera und schweren Kontaktallergien. Teils deutliche Beläge mit Juckreiz und Rötungen seit 2 Monaten. Ansicht linker Unterschenkel von innen. Klinischer Verdacht auf Tinea pedis und Onychomykose, der jedoch mehrfach zytologisch nicht erhärtet werden konnte. In den Belägen der Unterschenkelhaut Hyphennester.

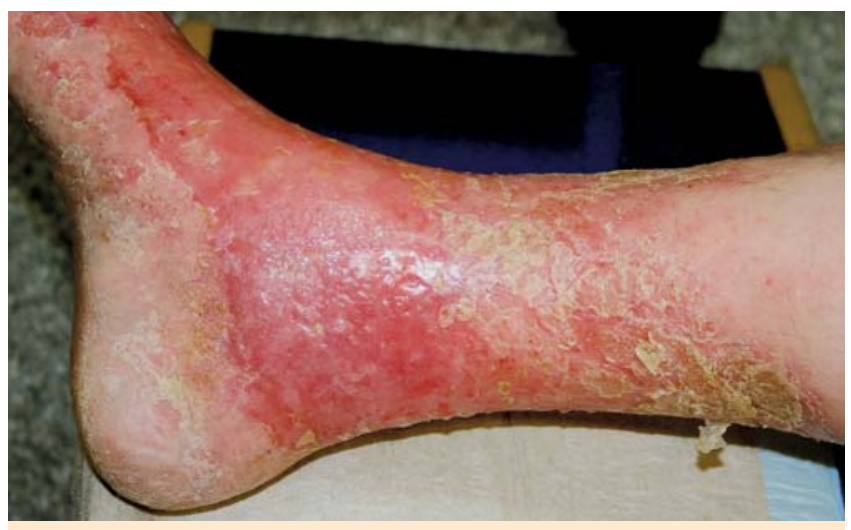

Abb. 11 Gleiche Patientin wie in $\bullet$ Abb. 10. Ansicht des rechten Innenknöchels: Erosiv nässende Stauungsdermatose mit Nestern aus Hyphenfäden. Lokale Antimykose wegen der Kontaktallergien mit Clotrimazol 2\% in Vaseline flavum. Hier sei nochmals betont, dass alle Patienten routinemäßig mit dem antimykotisch ausgewiesenen Wundantiseptikum Octenidin-Lösung vorbehandelt wurden. Deutliche Besserung der Befunde innerhalb von $1-2$ Wochen.

zuhalten. Eine sichere Diagnostik ist dabei nur wegen der bekannten Nachteile der Kulturdiagnostik (Nichtanwachsen, Verschimmelung, multiples Keimspektrum, Sekundärverkeimung, Parasiten usw.) durch zytologische bzw. histologische Auswertungen zu erzielen, da hiermit auch sehr geringe Pilzmengen erkennbar werden und der Pilz in seiner realen Ausbreitung auf der Haut und im Belag aktuell analysiert werden kann. Als erste zytologische Färbung sollte Methylenblau eingesetzt werden, die meist einen sehr guten und aussagekräftigen, panoptischen Überblick ermöglicht. Danach, wenn sich keine Pilzelemente zeigen, sollten Gramfärbungen und dann PAS- oder Fluoreszenzanalysen erfolgen. Bei dickeren Belägen sollten parallel zur Zytologie auch histologische Kontrollen erfolgen ( $\bullet$ Abb. 1, 2, 10, 11). Die zytologische Suche wird oft durch die lokale Therapie erheblich erschwert. Salbenreste, Zellstofffasern, Verbandsmullfäden, Hydrogele u.a.m. können sich auf der Haut zu dichten Belägen zusammenfügen, die die farblichen Markierungen der Pilzstrukturen deutlich weniger effektiv machen und sogar zu negativen Kontrastphänomenen führen (ähnlich Probleme wie bei der bakteriologischen Wundgrundzytologie [3]). Auch aus diesen Gründen sollten unbedingt mehrere zytologische Proben entnommen werden, um in verschiedenen Schichten und Milieuverhältnis- sen am Unterschenkel und in der Wunde die Pilze sicher detektieren zu können. Dickere Beläge sollten mit einer Pinzette abgehoben und abgeschnitten werden, um dann zytologisch aufbereitet zu werden. Die Nutzung der Fluoreszenz-Technik, die noch kombiniert werden kann mit mykologischen GensondenMarkierungen, würde diese zytologischen Analysen erheblich erleichtern.

Besonders sind Patienten von mykotischen Nachbarschaftsinfektionen bedroht, deren Unterschenkelhaut erosiv und nässend ist ( Abb. 1, 2, 10, 11). Dies trifft auf viele Patienten mit einem chronisch venös-lymphatischen Stauungsekzem und erosiven Lymphödemen zu. Diese Milieuverhältnisse sind bekannte, ideale Voraussetzungen für kritische bzw. gefährliche bakterielle Infektionen, aber auch wie hier beschrieben für schwere mykotische Begleitinfektionen.

Wir haben gelernt, auf bakterielle Problemkeime in Belägen und Exsudaten der Wunden zu achten und diese zu behandeln. Wir sollten aber ebenso auch an mykotische Probleminfektionen der vorgeschädigten Unterschenkelhaut denken, vor allem wenn diese Beläge aufweisen. Diese existieren meist unbemerkt hoch chronisch über Monate und Jahre in der Haut, verursachen chronische Hautschäden und komplizieren noch weiter das aufwendige Therapie- und Wundmanagement ( Abb. 1, 2). Das venös bedingte Ulkus mit seiner umgebenden Problemhaut wird klinisch völlig atypisch von den Pilzen über lange Zeit hinweg infiltriert und durch gut gemeinte und sonst sinnvolle Therapien wie Hydrokolloidverbände oder lokale Kortisonanwendungen wegen des Verdachtes auf kontaktallergische Hautreaktionen noch weiter gefördert ( $\bullet$ Abb. 1, 2). Wie in meiner Fallsammlung deutlich zu erkennen war, reicht die alleinige lokale Behandlung der Unterschenkel und Füße mit dem verwendeten Antisepticum nicht aus, obwohl dieses nachweislich antimykotisch wirksam sei (siehe Produktinformation der Fa. Schülke \& Mayr GmbH). Alle beschriebenen Patienten wurden routinemäßig bei jeder Behandlung mehrfach pro Woche mit OctenidinLösung behandelt.

Diese sinnvolle Therapie muss nach dem Nachweis einer mykotischen Unterschenkel-Infektion durch breitbandantimykotisch wirksame Lokaltherapeutika ergänzt werden. Hierbei ist auch der gesamte Fuß mit den Zehenzwischenräumen und Zehenbeeren einzucremen.

Sinnvoll können auch Lokaltherapien mit Kombinationen von modernen Azol- und Terbinafin-Präparaten [5] sein.

Es ist unbestritten, dass die humanrelevanten Pilze, vor allem die Hefepilze, im feuchtwarmen und dunkeln Milieu ideal wachsen [1,2,4-8]. Gerade aber diese Milieuverhältnisse sind es, die bei der Anwendung des modernen Wundmanagements herrschen. Der definiert feuchte Wundverband, der über mehrere Tage hinweg unter den lichtgeschützten Verhältnissen darüber gewickelter Kompressionsverbände und der äußeren Beinbekleidung angelegt wurde, stellt die therapeutisch gewollten, bewährten Heilungsbedingungen zur Wundregeneration dar, die aber auch umgekehrt ideale Lebens- und Wachstumsbedingungen für Bakterien und Pilze darstellen können. Der Autor kontrolliert deshalb routinemäßig bei längeren Verbandtragezeiten und nach den Praxisurlauben die Wunden und deren Umgebung zytologisch auf deren bakteriellen und mykotischen Status (siehe hier die Erstveröffentlichung der Wundgrundzytologie [3]). Die großen Wundpflegefirmen haben ihren Fokus bisher vor allem auf die Eindämmung der bakteriellen Gefährdung chronischer Wunden gelegt. Mit dieser Publikation ist die Anregung verbunden, nachweislich in-vivo-antimykotisch wirksame 


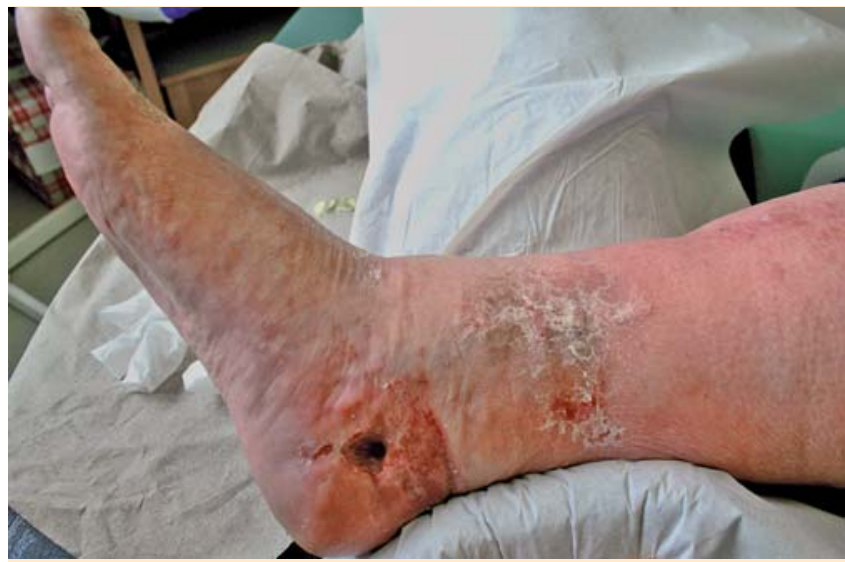

Abb. 12 73-jähriger, übergewichtiger Patient mit pAVK und Z.n. mehrfachen Bypaß-Operationen. Durch die Gefäßentnahmen am rechten Unterschenkel kam es zu einem postoperativen Lymphödem und massiven Schmerzparaesthesien am Vorderfuss und den Zehen.

Diese Schmerzen behinderten stark die Behandlung des Fußes. Beläge am distalen Unterschenkel. Juckreiz und Rötung.

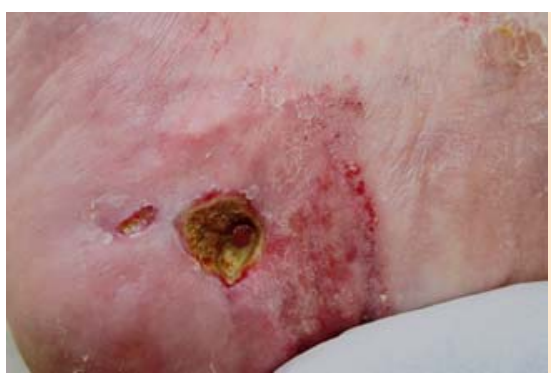

Abb. 13 Detail der chronischen Wunde im Innenknöchelbereich oberhalb der Ferse. In der Wundumgebung wurden Hyphen gefunden.

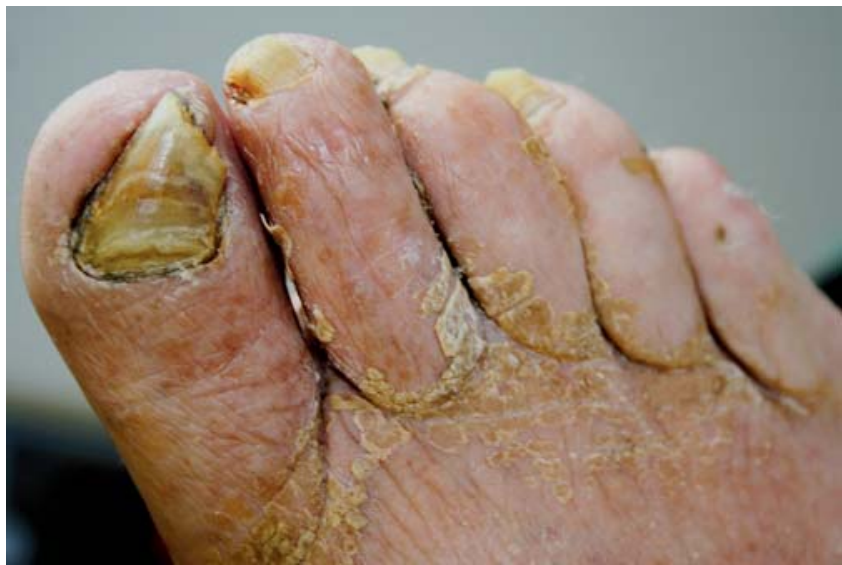

Abb. 14 Patient aus Abb. 12 u. Abb. 13 mit massiver Onychomykose und Tinea interdigitalis. Antimykotische Lokaltherapie mit CiclopiroxCreme und kurzzeitig oral mit Terbinafin.

Wundauflagen bzw. Wundtherapeutika zu entwickeln. Zudem sollten fertige antimykotische Lokaltherapeutika oder variable antimykotische Rezepturen nutzbar sein, die nur ein geringes allergisierendes Potenzial aufweisen.

Es sei noch ein weiterer wichtiger Sachverhalt hierzu erwähnt. Die Pilze schädigen durch ihre Enzymausstattung, Toxine und das Allergenisierungspotenzial noch weiter die schon vorgeschädigte Haut des Unterschenkels. Sie verstärken die Entzündung in der Stauungsdermatitis und vermindern weiter die Barrierefunktion und Abwehr in der Unterschenkelhaut ( $\bullet$ Abb. 1,
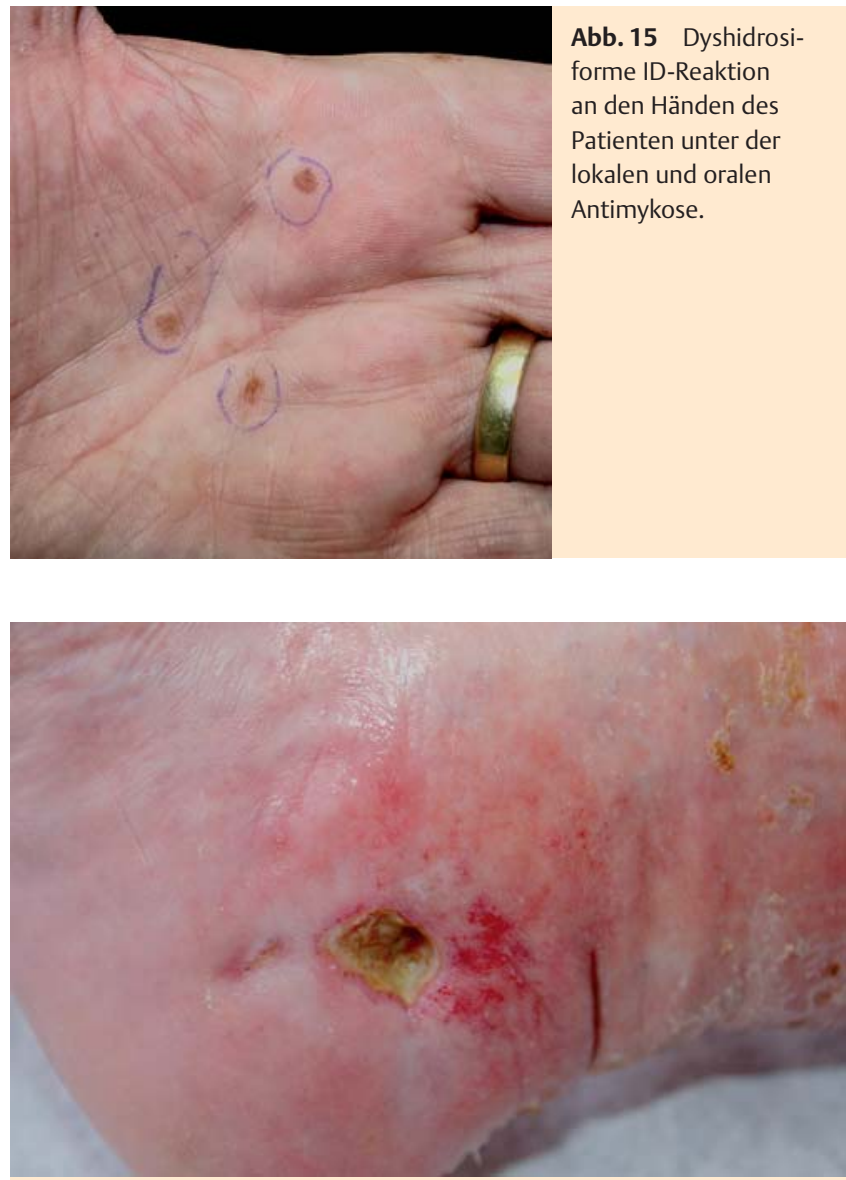

Abb. 16 Deutliche Verkleinerung und Abflachung des Ulkus 2 Wochen nach intensiver externer und nur kurzer interner Antimykose mit Terbinafin. Abbruch der oralen Antimykose wegen erheblicher ID-Reaktion.

2, 10, 11). Diese Umstände begünstigen ihrerseits wieder das Eindringen pathogener Keime in die Stauungsdermatose und fördern das chronische Erysipel. Der Basisschaden der Stauungsdermatose, vor allem bei erosiv-nässenden Hautverhältnissen, fördert die Invasivität der normalerweise oberflächlichen Tinea. Die Chronizität und Rezidivfähigkeit dieser atypischen Tinea cruris ist sehr gut vergleichbar mit den Gegebenheiten bei der chronischen und maskierten Tinea pedis. Die chronische Pilzinfektion erleichtert die akute und chronische bakterielle Kolonisation und Infektion, wie in den Zehenzwischenräumen seit langem bekannt. Eine mykotische Milieusanierung hilft deshalb auch in der Vorbeugung bakterieller Komplikationen.

Bei der Behandlung chronischer Wunden und deren vorgeschädigter Hautumgebung müssen Dermatologen einbezogen werden. Die Arbeit zeigt die Vorteile der zytologischen Vorortdiagnostik im Praxislabor zusammen mit dem klinischen Beurteilungsvermögen deutlich. Die Bakteriologie und kulturelle Mykologie in den Laboren kann mit Fehlerquellen verbunden sein, die die Vorortzytologie nicht hat. Mit der schnell auswertbaren Zytologie kann sofort der mykologische Hautstatus abgeklärt und einfach in zweckmäßigen Abständen unter der Therapie überprüft werden.

Diese Publikation beschreibt die Infektionsgefahren durch benachbarte Pilzinfektionen der vorgeschädigten Unterschenkelhaut und deren chronischen Wunden. In der relevanten mykologischen Fachliteratur [1,2,4-6,8] waren keine Fakten hierüber zu finden. Ich halte hier den eigenständigen Begriff der pedocru- 
ralen Tinea als Nachbarschaftsmykose bzw. als mykotische Infektionseinheit für gerechtfertigt, weil dieser deutlich macht, dass diese Körperareale zusammen mykotisch abgeklärt und behandelt werden müssen sowie sich gegenseitig infizieren können. Außerdem hat diese Tinea die Eigenart, dass ihr Erscheinungsbild am Unterschenkel nicht den üblichen klinischen Bildern einer Tinea corporis entspricht, sondern weitgehend überformt und überlagert wird von den chronischen Vorschäden des Unterschenkels ( Abb. 1, 2, 5, 6, 10-13). Diese Tineaform frisst sich vor allem unter dem klinischen Bild der erosiven oder entzündlichen Stauungsdermatose langsam, hoch chronisch und maskiert, über Jahre hindurch und diskontinuierlich in die schutzlose Haut des Unterschenkels. Wird diese Tinea jedoch erkannt und konsequent behandelt, so zeigt sich innerhalb weniger Tage die Besserung des Hautzustandes. Die wahren Beschwerden der Stauungsdermatosen und Ulzerationen bleiben übrig oder bessern sich oft synchron mit der antimykotischen Therapie.

Es ist seit Jahrzehnten bekannt, dass Patienten mit chronisch venösen Stauungsdermatosen deutlich häufiger eine Tinea pedis und Onychomykose aufweisen [4]. Dies hatte sich auch in dieser Fallsammlung gezeigt. Daraus folgt die Empfehlung, dass jeder crurale Wundpatient mit einer Onychomykose oder Tinea pedis genau und wenn nötig mehrfach bzgl. einer mykotischen Nachbarschaftsmykose abgeklärt werden muss, auch wenn keine Beläge vorhanden sind.

\section{Abstract}

\section{Pedocrural Tinea in Previously Damaged Lower Leg Skin or Leg Ulcers}

$\nabla$

This manuscript focuses on how onychomycosis, interdigital mycosis of the feet or tinea pedis compromises the treatment of chronic venous and lymphatic stasis dermatitis.

Tinea pedis, interdigital mycosis of the feet, and onychomycosis often lead to direct mycotic infection of previously damaged skin on the lower leg and chronic wounds in the proximity. This tinea cruris and wound mycosis are not the typical presentation of tinea corporis because of the underlying damage and therefore difficult to identify (tinea incognita). The ideal environment for this local mycosis is especially the chronic venous and lymphatic leg ulcer with skin erosion by a weeping wound. The diagnosis should be confirmed cytologically, or histologically by taking curettage samples.

Intensive topical antimycotic treatment must encompass the entire foot and lower leg as a mycotic infectious unit (pedocrural tinea). Consistent topical antimycotic treatment will improve the condition of the skin on the lower leg within a few days and facilitate modern wound management. Already-damaged skin on the lower leg should be treated prophylactically for mycosis in the presence of onychomycosis or tinea pedis.

\section{Literatur}

1 Braun-Falco $O$ et al. Dermatologie und Venerologie, 5. Auflage. Heidelberg: Springer, 2005

2 Grigoriu D, Delacretaz J, Borelli D. Lehrbuch der medizinischen Mykologie. Bern: Hans Huber, 1984

3 Foss P. Die Wundgrundzytologie. Akt Dermatol 2008; 34: 262 - 265

4 Fritsch P. Dermatologie, 3. Auflage. Heidelberg: Springer, 1990

5 Hof H. Mykologie für Mediziner. Stuttgart: Thieme, 2003

6 Salfelder K. Pilzinfektionen beim Menschen. Hamburg: OmniMed, 2000

7 Seebacher C, Blaschke-Hellmessen R. Für die medizinische Praxis, Mykosen. Jena: Gustav Fischer, 1990

8 Seeliger HPR, Heymer T. Diagnostik pathogener Pilze des Menschen und seiner Umwelt. Stuttgart: Thieme, 1981 